\title{
Inhalt
}

Schwerpunktthema:

Kritische Lebenskunst

Möglichkeiten und Grenzen

eines gelingenden Lebens

Alltägliche, gesellschaftliche

und therapeutische Kontexte

Editorial

GÜNTER GÖDDE, JÖRg ZIRFAS

\& HANS-JÜRGEN WIRTH

Lebenskunst in der Therapie

Ein Fallbeispiel

GÜNTER GöDdE \& Jörg Zirfas

Rechtfertigung, Abschaffung, Bejahung

Wege zum Umgang mit Leiden

Helmut Heit

Ungerechtigkeit und Missachtung

Über Erwartungen und Erfahrungen

JöRG ZIRFAS

Echt hässlich!

Über das Nicht-Schöne

in Kunst, Kultur und Psyche

Karin DANNecker

Alltagskunst

Zu einer Psychästhetik des Alltags

Werner Pohlmann

Offensiv leben

Über das Scheitern als gesuchte Chance

ROBERT ZIMMER

Lebenskunst und Narzissmus

Schwierigkeiten und Chancen

des selbstreflexiven Selbst,

das Leben zu meistern

HANS-JÜRGEN WIRTH
Liebe und Sehnsucht

Bedrohungen und Möglichkeiten der Liebe in modernen Zeiten

Silvia SCHNeIDER

5

»Die Zeit heilt alle Wunden «

90

$\mathrm{Zu}$ einem Spruch der (kritischen) Lebenskunst

Werner Stegmaier

Erinnern und Vergessen

in der therapeutischen Lebenskunst

11 GüNTER GöDDE

Psychotherapie und kritische Lebenskunst aus Sicht der Therapieforschung

23 Heinrich Deserno

\section{Freier Beitrag}

33 Überlegungen zu Musik unter Verweis auf ausgewählte Modelle psychischer Gesundheit

ERIC Pfeifer

42

\section{Rezensionen}

(Selbst-)Kritik und Krisenhilfe

Günter Gödde \& Jörg Zirfas (Hrsg.). (2018).

53 Kritische Lebenskunst. Analysen -

Orientierungen - Strategien

Dagmar Kiesel

62 Anmut, Würde, Leidenschaft -

Lebenskunst revisited

Günter Gödde \& Jörg Zirfas (Hrsg.). (2018).

Kritische Lebenskunst. Analysen -

71 Orientierungen-Strategien

Hans-Gerd von Seggern

Dank an die Gutachterinnen

und Gutachter

Impressum 


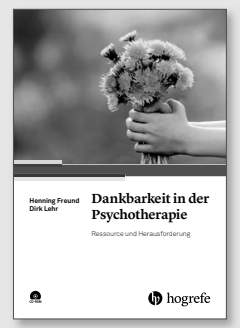

Henning Freund/

Dirk Lehr

Dankbarkeit in der

Psychotherapie

Ressource und

Herausforderung
2020, 217 Seiten, inkl. CD-ROM,

$€ 29,95 /$ CHF 39.90

ISBN 978-3-8017-2893-9

Auch als eBook erhältlich

Das Thema Dankbarkeit erhält in der Psychologie zunehmend Aufmerksamkeit. Viele Studien belegen die hohe Relevanz von Dankbarkeit für die psychische Gesundheit. Das Buch informiert umfassend über den aktuellen Wissensstand rund um Dankbarkeit und stellt zahlreiche Übungen sowie Arbeitsmaterialien zur Förderung von Dankbarkeit vor.

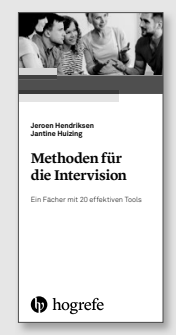

Jeroen Hendriksen/

Jantine Huizing

Methoden für die Intervision

Ein Fächer mit

20 effektiven Tools

2020, 72 Seiten, Kleinformat,

$€ 16,95 /$ CHF 21.90

ISBN 978-3-8017-3033-8

Für die Arbeit in Intervisionsgruppen stellt dieser Fächer zwanzig effektive Arbeitsmethoden vor. Unterschieden werden dabei lösungsorientierte, kreative, aktivierende und reflexive Arbeitsformen. Die vorgestellten Tools geben neue Impulse für die Intervision.

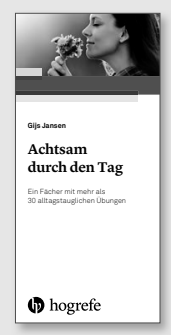

Gijs Jansen

Achtsam durch den Tag

Ein Fächer mit mehr als

30 alltagstauglichen

Übungen
2020, 54 Seiten, Kleinformat,

$€ 16,95 /$ CHF 21.80

ISBN 978-3-8017-3034-5

Dieser Fächer ist ein praktischer Begleiter für alle, die Achtsamkeit praktizieren und sich selbst und ihre Umgebung neu erkunden wollen. Er enthält mehr als 30 Übungen für den Alltag. Achtsamkeit ist keine Frage der Örtlichkeit, Sie können immer und überall achtsam sein. Mit den Übungen lernen Sie vor allem, Ihre Wahrnehmung zu schärfen und offen zu sein für das, was sich im Hier und Jetzt ereignet.

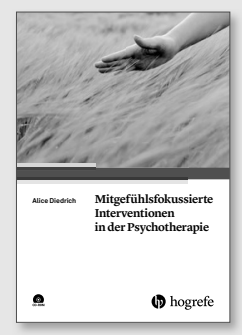

\section{Alice Diedrich \\ Mitgefühls- \\ fokussierte \\ Interventionen in der Psychotherapie}

2016, VIII/152 Seiten, inkl. CD-ROM, $€ 26,95 / \mathrm{CHF} 35.90$

ISBN 978-3-8017-2671-3

Auch als eBook erhältlich

Das Buch stellt Interventionen zur Steigerung von Mitgefühl in der psychotherapeutischen Praxis vor. Übungsanleitungen, Fallbeispiele und Patient-Therapeut-Dialoge illustrieren das Vorgehen.

www.hogrefe.com 
Schwerpunktthema:

\section{Kritische Lebenskunst}

Herausgegeben von Günter Gödde, Jörg Zirfas und Hans-Jürgen Wirth 


\section{Kritische Lebenskunst}

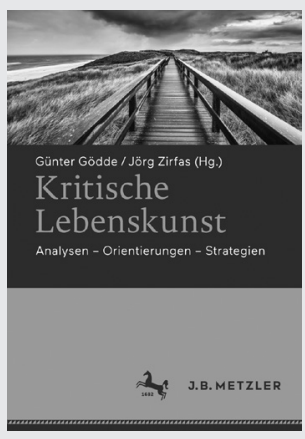

G. Gödde, J. Zirfas (Hrsg.)

\section{Kritische Lebenskunst}

Analysen - Orientierungen - Strategien 2018, XIV, 490 S. 10 Abb., 7 Abb. in Farbe. Geb. $€(D) 49,99$ | $€(A) 51,39 \mid{ }^{*} \mathrm{CHF} 55.50$

ISBN 978-3-476-04643-7

$\rightarrow$ Geschrieben von Expert/innen aus Theorie und Praxis

$\rightarrow$ Sozial und kulturell fokussierte Lebenskunst

$\rightarrow$ Interdisziplinäre Behandlung des Themas

Die Philosophie der Lebenskunst gehört seit Ende des 20. Jahrhunderts zu den aktuellen Denkrichtungen, die nicht nur in Lehre und Forschung vertreten, sondern auch von einem breiteren Publikum wahrgenommen werden. In ihr geht es um ein gelungenes, schönes und glückliches Leben.

$€(D)$ : gebundener Ladenpreis in Deutschland, $€(A)$ : in Österreich. * unverbindliche Preisempfehlung. Alle Preise inkl. MwSt.

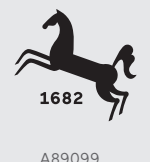

\section{鸪 Psychosozial-Verlag}

Günter Gödde \& Jörg Zirfas

\section{Therapeutik und Lebenskunst}

Eine psychologisch-philosophische Grundlegung

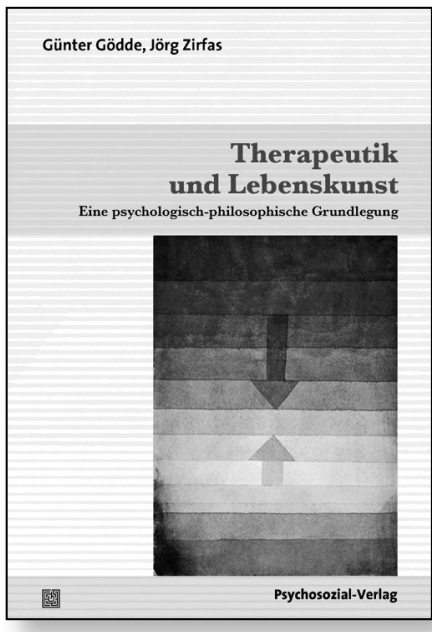

733 Seiten $•$ Broschur $\bullet € 69,90$ ISBN 978-3-8379-2439-8

Therapeutik und Lebenskunst sind in vielfältiger Weise miteinander verknüpft. Lebenskunst ist in existenziellen Problemlagen auf therapeutische Erfahrungen und Praktiken angewiesen; umgekehrt sollte Psychotherapie das Deutungs-, Orientierungs- und Reflexionswissen der Lebenskunst heranziehen. Lebenskunst ohne Psychotherapie stellt eine praktische und Psychotherapie ohne Lebenskunst eine theoretische Verkürzung dar.

Günter Gödde und Jörg Zirfas beleuchten die zentralen Dimensionen der philosophischen und therapeutischen Lebenskunst und erörtern deren Bedeutung für die psychotherapeutische Praxis.

\section{Walltorstr. $10 \cdot 35390$ Gießen \\ Tel. 0641-969978-18 \\ Fax 0641-969978-19 \\ bestellung@psychosozial-verlag.de www.psychosozial-verlag.de}




\title{
Möglichkeiten und Grenzen eines gelingenden Lebens
}

\section{Alltägliche, gesellschaftliche und therapeutische Kontexte}

\section{Editorial}

\author{
Günter Gödde, Jörg Zirfas \& Hans-Jürgen Wirth
}

\author{
psychosozial 43. Jg. (2020) Heft IV (Nr. 162) 5-10 \\ https://doi.org/10.30820/0171-3434-2020-4-5 \\ www.psychosozial-verlag.de/ps
}

Lebenskunst hat Konjunktur. Dafür gibt es eine Reihe von Gründen, die mit den Entwicklungen und Problemlagen der Moderne zu tun haben. Stichwortartig kann man hier etwa nennen: die aktuellen kulturellen Verunsicherungen angesichts von Migrationsbewegungen und Globalisierungsentwicklungen, die Bedrohungen durch das Corona-Virus und den internationalen Terrorismus, die ökonomischen Ängste vor dem Abstieg, dem Prekariat oder der Exklusion, die populistischen Debatten um Identitäten und Verluste, die Infragestellung des (wissenschaftlichen) Wissens durch fake news, die Entwicklungen im religiösen Verhalten weg von den konfessionellen Kirchen und hin zu spirituellen und parapsychologischen Bewegungen, die Erfahrungen von Müdigkeit und Depression angesichts einerseits potenziell unendlich vieler Wahl- und Entscheidungsspielräume und andererseits potenziell wachsender Verantwortlichkeiten, die Enttäuschungen angesichts sozialer Erosionen und Spaltungen der Gesellschaft und nicht zuletzt die atomare Bedrohung angesichts von 28.000 Atomwaffen und die ökologische Bedrohung angesichts der Klimakatastrophe. Anders und kurz formuliert: Lebenskunst ist immer dann gefragt, wenn man sich nicht mehr auskennt und nicht mehr weiß, wie es weitergehen soll. Die Frage nach der $»$ Lebenskunst « wird in Zeiten virulent, wenn sich das Leben immer weniger von selbst versteht, wenn Traditionen, Konventionen und Normen an Überzeugungs- kraft verlieren und die Individuen sich um sich selbst zu sorgen beginnen. Dabei hat die Lebenskunst im Kern ein praktisches Ziel, geht es ihr doch letztlich nicht um die große Theorie einer magna moralia, sondern um die kleine Praxis einer ars vivendi, die auch und gerade auf den Alltag der Menschen zielt. Und dieser Alltag soll letztlich den Kriterien eines geglückten und schönen, das heißt eines bejahenswerten Lebens gerecht werden.

Auch die diversen Psychotherapien, als Schulen der Lebenskunst betrachtet, beziehen sich auf die Suche nach dem »richtigen Leben « und eine diesbezügliche »Formung « der Menschen. Dabei geht die therapeutische Lebenskunst zunächst von einer negativen Grundannahme aus, nämlich dass Menschen aus vielerlei Gründen mit ihrem Leben nicht »zurechtkommen « und dass wir es dabei mit häufig schwierigen, existenziellen Leidenserfahrungen zu tun haben. In diesem Sinne lässt sich die These aufstellen: Lebenskunst zielt, wenn es um Fragen von existenzieller Bedeutsamkeit geht, auf eine Form der (Psycho-)Therapie. Lebenskunst ist dann auf »Therapie« (in einem weit gefassten Sinne) angewiesen, wenn die psychischen Probleme schwerwiegender, intensiver, umfänglicher und folgenreicher sind, als dass man sie allein mit Selbstreflexion und Selbstbildung oder auch mit Gespräch und Beratung bewältigen könnte. Psychotherapeutische Einsätze erscheinen aber auch dann sinnvoll, wenn sie uns über die unbewusst gelingenden Mechanismen des Alltags aufklären. Denn Men- 
schen kommen ja durchaus in unterschiedlichem Ausmaß mit ihrem Leben zurecht - auch wenn sie dieses »Gelingen « nicht immer reflektieren, strukturieren und konsequent und intensiv betreiben. Psychotherapien helfen insofern auch, als sie auf gelingende Formungs- und Strukturierungsprinzipien und -praktiken des Alltags hinweisen, ohne daraus allumfassende und allgemeingültige Ansprüche ableiten zu wollen.

In ihrem Buch Therapeutik und Lebenskunst (2016) gehen Günter Gödde und Jörg Zirfas davon aus, dass die vielfältigen, von der Antike bis in die Moderne reichenden Zusammenhänge zwischen therapeutischen Theorien und Praktiken und Überlegungen zur Lebenskunst in den aktuellen Debatten kaum thematisiert werden. Diese vergessenen Zusammenhänge kann man mit Blick auf die Lebenskünste ebenso konstatieren, da diese ihre therapeutischen Dimensionen vernachlässigt haben, wie für die Therapiemodelle, die sich ihre »impliziten Konzepte « der Lebenskunst in der Regel nicht bewusst gemacht haben. Denn die aktuellen Debatten in der Lebenskunst sind zentriert um Selbstreflexion und gemeinsame Beratung, weniger um Selbstbehandlung und therapeutisches Setting; und die aktuellen Debatten in der Psychotherapie betreffen eher die Diagnosen von $»$ Störungen « sowie technologische Erklärungs- und Behandlungsmodelle als den Umgang mit existenziellen Problematiken, kulturellen, ökonomischen und politischen Rahmenbedingungen und der Praxis einer modernen gelingenden Lebenskunst.

Inwiefern ist eine therapeutische Lebenskunst kritisch? Die Herausgeber gehen davon aus, dass sich die Kritik einer therapeutischen Lebenskunst auf verschiedene Ebenen beziehen kann, von denen einige schon in der Kritischen Lebenskunst (Gödde \& Zirfas, 2018) entfaltet wurden: Sie kann sich auf therapeutische Theorien und Modelle und ihre Rationalitäten und Ideologien beziehen, die sich aus Sicht einer pragmatischen Lebenskunst als elitär, unangemessen und/oder nicht sinnvoll darstellen; sie kann sich auf sinnlich-leibliche Zusammenhänge oder auf vielfältige Erfahrungen konzentrieren, in denen sich Menschen als ohnmächtig oder als (extrem) abhängig erleben; sie kann sich sozial- und kulturkritisch äußern, indem sie soziale Ungleichheiten, politische Dominanzverhältnisse oder kapitalistische Steigerungslogiken und damit auch eine gelungene Lebenskunst infrage stellt und nicht zuletzt kann sie sich auf ein therapeutisches Verhalten der Therapeut*innen selbst beziehen, in denen unangemessene Formen der Lebenskunst ihren Ausdruck finden.

Eine kritisch-therapeutische Lebenskunst zielt nicht auf das große Glück des Lebens, sondern auf die kleinen und unscheinbaren Sublimierungen und Stilisierungen des Alltags. Im Zentrum stehen dabei einerseits die Möglichkeiten, mit den Krisen- und Unglückssituationen umgehen zu können. Dabei erscheint wohl weniger bedeutsam, sein Leben als ästhetisch gestaltetes Kunstwerk zu verstehen, sondern viel wichtiger ist, wie man mit biografischen Brüchen und Schicksalsschlägen umgehen kann. Und andererseits stehen dabei die Möglichkeiten im Blick, durch welche Praktiken, Rituale, Formen und Gestaltungen Menschen einen »gelingenden « Alltag bzw. eine bejahenswerte Existenz herstellen können. Denn jedes Leben hat schon eine Gestalt oder eine Form, die die Einzelnen durch ihr Leben »trägt«; es hat eine Struktur, die sich einer Strukturierung verdankt, die von unterschiedlichen Akteuren geprägt ist - vom Subjekt selbst, aber auch von seinen sozialen Beziehungen, von der Wirtschaft, der Kultur oder auch von den Medien.

Insofern soll der vorliegende Band Alltagsphänomene wie etwa Leiden, Zeitlichkeit, Liebe und Offensivität im Hinblick auf ihre existenziellen Tiefen wie auf ihre therapeutischen Anschlussmöglichkeiten aus unterschiedlichen Disziplinen in den Blick nehmen, um mithilfe der Lebenskunst und der Therapie kritische Überlegungen und pragmatische Vorschläge zum Umgang mit ihnen zu entfalten.

\section{Zu den Beiträgen in diesem Heft}

Das Heft wird mit einem Aufsatz von Günter Gödde und Jörg Zirfas eröffnet, der einen fallorientierten Einstieg in die Thematik einer Kritischen Lebenskunst liefern soll. Anhand eines Fallbeispiels, der Therapie eines verunsi- 
cherten und depressiven 30-jährigen Patienten, werden lebenskunstorientierte therapeutische Entwicklungs- und Lernprozesse rekonstruiert. Dabei werden fünf Stufen idealtypisch benannt, die von den aktuellen Problemen des Patienten und seiner Entwicklung in Kindheit und Jugend über die in der Therapie möglich werdende Erweiterung von Wahrnehmungsmöglichkeiten und Handlungsoptionen bis hin zur Umsetzung dieser Optionen in die Lebenspraxis und zur Herausarbeitung eines neuen Lebensentwurfs reichen. In diesem Prozess hat eine kritische therapeutische Lebenskunst nicht nur die biografischen Erfahrungen und Erschütterungen, sondern auch die sozialen, kulturellen und ökonomischen Zumutungen zu berücksichtigen, die mit den modernen Forderungen nach Glück, Erfolg und Selbstverwirklichung verbunden sind.

Dass eine kritische Philosophie der Lebenskunst zentral mit dem Thema des Leidens, der Enttäuschungen und Krisen umgehen muss, zeigen die philosophischen Analysen von $\mathrm{Hel}$ mut Heit. Er arbeitet mit Blick auf die Geschichte der abendländischen Philosophie exemplarisch mit Platon, Karl Marx und Friedrich Nietzsche drei je unterschiedliche Auffassungen heraus, sich zum Leiden zu verhalten: Platon bemüht sich über den Weg der Rechtfertigung, dem Leiden mithilfe von Religion oder Philosophie einen Sinn zu verleihen; eine Perspektive, die dann auch im Christentum und insbesondere bei Blaise Pascal eine wichtige Rolle spielen wird. Der Weg der Abschaffung, den Marx vorschlägt, bekämpft die Ursachen und Wirkungen des Leidens vor allem mit Wissenschaft und Technik. Nietzsche versucht dagegen den Weg der Affirmation oder Bejahung des Leidens zu gehen, indem er das Leiden als liebenswerten Teil des Lebens anerkennt, wobei er zwischen nötigem und unnötigem Leiden unterscheidet. Heit verweist zudem darauf, dass eine philosophische Behandlung des Leidens nur dann möglich ist, wenn spezifische Schmerzgrenzen akut nicht überschritten werden.

Im Text von Jörg Zirfas über die Erfahrungen von Ungerechtigkeit wird aus einer sozialwissenschaftlichen und philosophischen Per- spektive zunächst deren zunehmende Bedeutung in der Moderne in den Blick genommen. Diese ist dadurch bedingt, dass Ungerechtigkeit als Verletzung der Selbstachtung und somit als Erfahrung der Missachtung und Diskriminierung bzw. als Beschädigung der physischen, sozialen und moralischen Integrität verstanden wird. Aus Sicht einer Kritischen Lebenskunst gilt es, die Perspektive von individuellen Erfahrungen von Ungerechtigkeit konsequent ernst $\mathrm{zu}$ nehmen. Um Ungerechtigkeiten zu begegnen, braucht es einerseits einen individuellen Sinn für Ungerechtigkeit und andererseits politische Verfahren, die alle Betroffenen so weit wie möglich an der Aufstellung gerechter demokratischer Prinzipien und Verfahren beteiligen. Die Kritische Lebenskunst verfolgt das Ziel, die Differenz zwischen den persönlichen Erwartungen von Gerechtigkeit und den Erfahrungen von Ungerechtigkeit soweit als möglich $\mathrm{zu}$ verringern.

In ihrem Betrag nähert sich Karin Dannecker einem Thema, mit dem sich kaum jemand gerne beschäftigt: dem Hässlichen. Die Konfrontation mit etwas Hässlichem weckt Gefühle des Unbehagens, der Angst, des Ekels oder der Verachtung. Die Wirkung auf die Sinne und das ästhetische Empfinden kann extrem verstörend und folgenreich sein, denn die Erfahrung mit Hässlichkeit aktiviert oft unbewusst Fantasien über die eigene Fragilität und Vergänglichkeit. An Beispielen aus der bildenden Kunst und der Kunsttherapie untersucht die Autorin, was unter ästhetischen Gesichtspunkten als hässlich gilt, und wie die intensiven emotionalen Reaktionen auf Hässlichkeit psychodynamisch zu erklären sind. Das Ergebnis verdeutlicht, dass sowohl im künstlerischen als auch im therapeutischen Prozess immer Elemente des Hässlichen enthalten sind. Sie bilden die Voraussetzungen für neue, transformierende Erfahrungen.

Werner Pohlmann arbeitet in seinem Beitrag heraus, dass sich eine Philosophie der Lebenskunst nur auf dem Boden einer »Alltagskunst « entwickeln kann. Anhand von psychologischen Untersuchungen zu Alltagsphänomenen wie Aufwachen, Sich-Spiegeln, SichKleiden und Aus-dem-Fenster-Schauen veranschaulicht der Autor die psychästhetische Lo- 
gik des Alltagsgeschehens. In einem zweiten Schritt wendet er sich dann dem Übergang von der Alltagskunst zur Erkenntnis »übergreifender Zusammenhänge « zu, wie sie von einer Philosophie der Lebenskunst gefasst werden. In mehreren Schritten lassen sich diese übergreifenden Zusammenhänge der Lebensgestaltung bewusst machen, indem Menschen sich ihre Lebensführung reflektierend zum Gegenstand machen. Auf diese Weise gewinnen sie ein Gespür dafür, wie sie in die Situation und in die Probleme geraten sind, die sie leiden lassen. Das ermöglicht es ihnen auch, sich darüber klar zu werden, welche unbewusste, paradoxe Konstruktion ihr Leben gleichsam wie ein universales Gesetz bestimmt.

Der philosophische Aufsatz von Robert Zimmer ist ein konsequentes Plädoyer für ein aktives Leben, das heißt ein bewusstes Leben, das auf die Welt zugeht und Stillstände überwindet. Seine Lebenskunst propagiert eine aktive Auseinandersetzung mit Fragen und Problemen des Lebens. Insofern lehnt er eine seit der Antike bis heute nachwirkende Tradition der Passivität und defensiven Lebenshaltung, die sich als Akzeptanz des scheinbar Unverfügbaren und als Vermeidungsstrategie gegenüber dem Scheitern äußert, dezidiert ab. Entscheidend für diese Lebenskunst ist eine Neubewertung des Scheiterns, das nicht mehr als zu vermeidendes Unglück, sondern als notwendige Korrekturinstanz eines besseren Lebens verstanden wird. Zimmer schlägt die Strategie einer kontrollierten Lebensoffensive vor, die nach dem Prinzip von Versuch und Irrtum verfährt und Mut und rationale Planung verbindet. Durch diese Form der Offensive werden Möglichkeiten eines experimentellen und autonomen Lebens eröffnet.

Hans-Jürgen Wirth betrachtet die Lebenskunst im Lichte der psychoanalytischen Narzissmustheorien. Narzisstische Störungen versteht er als eine Form des problematischen oder auch pathologischen Selbstbezugs, der einem gelingenden Leben im Wege steht. Man hat in der Kunst, sein Leben zu führen, viel erreicht, wenn es gelingt, einen Teil der allgegenwärtigen narzisstischen Kränkungen produktiv zu verarbeiten, indem man den seeli- schen Schmerz mentalisierend reguliert und Kränkungen in Selbsterkenntnis verwandelt. An gängigen Konzepten der philosophischen und der psychotherapeutischen Lebenskunst kritisiert Wirth die individualistische Ausrichtung und plädiert für die Praxis der »Zwiesprache « (Martin Buber) und der »Zwiegespräche« (Michael Lukas Moeller). Das psychoanalytische Konzept der Mentalisierung stellt er als theoretisch anspruchsvolles und gut erforschtes Modell für selbstreflexive Prozesse in der Lebenskunst dar.

Angesichts der individualisierten, beschleunigten und digitalisierten Welt der »flüchtigen Moderne «, in der Diskretion und Geheimnis zu verschwinden drohen, stellt Silvia Schneider Fragen nach den Bedrohungen für die Liebe und nach der Bedeutung der Sehnsucht für das moderne Subjekt. So ist auch nach dem Schicksal und der Bedeutung des Anderen, der Dauer, des Blicks und der Sprachen der Liebe zu fragen. Auf der Suche nach Antworten braucht es Literatur, Musik und Film, da die Liebe immer eine Narration oder ein Drama ist und die Wahrheit der Liebe eher erfahren als gewusst wird. Beispielsweise nimmt die Autorin anhand des Filmes Paterson von Jim Jarmusch die Liebe in ihrer Dauer in den Blick. Gezeigt werden soll, wie bedeutsam das Altägliche ist, wie die Liebe sich in der Dauer verwirklicht - im beharrlichen Ringen darum, Zwei-zu-Sein. So wird es für die Liebenden möglich, die Welt aus der Perspektive des Anderen immer wieder neu zu erschaffen.

»Die Zeit heilt alle Wunden« ist ein ebenso populärer wie plausibler Spruch der Lebenskunst - für Fälle schweren Leids, gegen das auch Psychotherapeut(inn)en nur wenig tun können. Von den falschen Leuten und zum falschen Zeitpunkt vorgebracht, kann er zynisch wirken. Denn die Zeit ist nichts, was etwas tun könnte. Wenn man dem Spruch jedoch wie Werner Stegmaier philosophisch nachgeht, so bekommt er einen guten Sinn. Dazu bedarf es eines kritischen Zeitbegriffs, den Augustinus auf den Weg gebracht hat und der hinter die messbare Zeit zurückgeht, und des modernen Begriffs der Orientierung. Sie ist so strukturiert, dass sich in ihr mit der Zeit Routinen einspielen, die 
ihr Halt geben und die, wenn sie einbrechen und Ängste, Verzweiflungen und Depressionen aufkommen, durch Routinen in anderen, weiterlaufenden Orientierungswelten kompensiert werden können. So, nicht durch bloßes Vergessen, kann wieder Orientierungssicherheit und mit ihr Zuversicht und neue Lebensfreude einkehren. Die Zeit hat die Wunden dann geheilt. Aber nichts zwingt zu glauben, dass sie das immer und immer gleichtäte.

In der Lebenskunstphilosophie der Antike wurden sowohl die »Kunst der Erinnerung «, die durch Mnemosyne (als Göttin des Gedächtnisses), als auch die »Kunst des Vergessens «, die durch Lethe (als Göttin des Vergessens) repräsentiert wurde, gepflegt. Therapeutisch gesehen geht es dabei in erster Linie um die Frage nach dem rechten Umgang mit unverarbeiteten Erfahrungen aus der Vergangenheit, aber auch grundlegender noch um den Wertkonflikt zwischen Erkennen und Leben. In Freuds therapeutischen Konzepten bildete das Erinnern, genauer gesagt: das Aufdecken »unbewusster Erinnerungen«, den Hauptfokus der psychoanalytischen Therapie. Bei Nietzsche hingegen stand das Vergessen im Blickpunkt, genauer gesagt: die Rehabilitierung der »aktiven Vergesslichkeit« als Form »starker Gesundheit«, ohne die es »kein Glück, keine Heiterkeit, keine Hoffnung, keinen Stolz, keine Gegenwart geben « könne. Statt Erinnern und Vergessen gegeneinander auszuspielen, sucht Günter Gödde sie in einem Zwei-Stufen-Modell für eine therapeutische Lebenskunst nutzbar zu machen. Im Rahmen psychodynamischer Psychotherapien kann das Erinnern-Können als erste Stufe und das Vergessen-Können als zweite Stufe in jedem therapeutischen Prozess betrachtet werden.

Heinrich Deserno rückt die Symbolbildung in den Mittelpunkt der Wechselwirkungen von Kritischer Lebenskunst und Psychotherapie. Die Arbeit oder das Handwerk des Lebens besteht in einer ständigen Balance von Polaritäten. Durch Symbolbildung werden Polaritäten vermittelt und überbrückt, zum Beispiel das Objektive mit dem Subjektiven. Während die Freud'sche Psychoanalyse den unbewussten Konflikt und wesentlich später das Trauma ins Zentrum ihrer Theorie gestellt hat, sind die der Philosophie entnommenen Polaritäten wie Paradoxien und Antinomien nicht nur Thema der Lebenskunst, sondern können auch der Logik der Psychotherapie zur Seite stehen. Symbole sind strukturbildend. In der psychischen Realität werden sie in unterschiedlicher Weise repräsentiert: als sensomotorisch-affektive Koordination, figurativ (bildhaft, sinnlich-symbolisch), konzeptuell (begrifflich, sprachlichsymbolisch) und in übergreifenden Diskursen. Abwehrmechanismen wirken als Desymbolisierung, die zum Beispiel als Klischee, Zeichenhaftigkeit und Somatisierung zu erkennen ist. Ein weiterer Schwerpunkt wird von Deserno auf die Möglichkeiten gelegt, die sich mit der Aufzeichnung und Transkription von Therapien für Forschung und Lehre ergeben.

Unser herzlicher Dank gilt den Autorinnen und Autoren dieses Themenheftes, denen das Anliegen und die Idee einer »Kritischen Lebenskunst« von Anfang an sehr eingeleuchtet hat und die sich in Ihren Beiträgen kompetent und kraftvoll dafür engagiert haben.

\section{Die Herausgeber}

Günter Gödde, Dr. phil., Dipl. Psych., jur. Assessor, psychologischer Psychotherapeut in eigener Praxis, Dozent, Supervisor, Lehrtherapeut und Ausbildungsleiter des Schwerpunkts Tiefenpsychologisch fundierte Psychotherapie an der Berliner Akademie für Psychotherapie und an der Psychologischen Hochschule Berlin. Ausgewählte Buchveröffentlichungen: Traditionslinien des »Unbewußten «. Schopenhauer, Nietzsche, Freud (2. Aufl. 2009); Das Unbewusste I-III (Hrsg. mit M. B. Buchholz 2005/06); Therapeutik und Lebenskunst (mit J. Zirfas 2016).

Jörg Zirfas, Prof. Dr. phil., Professor für Erziehungswissenschaft mit dem Schwerpunkt Pädagogische Anthropologie an der Universität zu Köln. Vorsitzender der Kommission Pädagogische Anthropologie (DGfE) und der Gesellschaft für Historische Anthropologie (FU Berlin); Mitglied des Interdisziplinären Zentrums Ästhetische Bildung (FAU ErlangenNürnberg), des Arbeitskreises Psychoanalyse und Lebenskunst (Berlin) und des Arbeitskreises Pädagogik und Vulnerabilität (Köln). Ausgewählte Buchveröffentlichungen: Schlüsselwerke der Vulnerabili- 
tätsforschung (mit D. Burghardt et al. 2019); Handbuch Bildungs- und Erziehungsphilosophie (Hrsg. mit G. Weiß 2019); Therapieziel Selbstsorge (mit G. Gödde 2021).

Hans-Jürgen Wirth, Prof. Dr. phil., Dipl.-Psych., ist Psychotherapeut, Psychoanalytiker und psychoanalytischer Paar-, Familientherapeut in eigener Praxis, Professor für Soziologie und Psychoanalytische Sozialpsychologie an der Universität Frankfurt/M. Er ist Gründer des Psychosozial-Verlags, Mitherausgeber der Zeitschriften psychosozial und Psychoanalytische Familientherapie. Ausgewählte Buchveröffentlichungen: Narzissmus und Macht (5. Aufl. 2015); Grenzerfahrungen. Migration, Flucht, Vertreibung und die deutschen Verhältnisse (Hrsg. mit R. Haubl 2019).

\section{Kontakt}

Dr. Günter Gödde

Kuno-Fischer-Str. 20

D-14057 Berlin

E-Mail: g.goedde@t-online.de

Prof. Dr. Jörg Zirfas

Universität zu Köln

Postfach 74, Gronewaldstraße 2

D-50931 Köln

E-Mail: joerg.zirfas@uni-koeln.de

Prof. Dr. Hans-Jürgen Wirth

Psychosozial-Verlag

Walltorstraße 10

D-35390 Gießen

E-Mail: hjw@psychosozial-verlag.de 